\title{
Some identities of Green's function for the polyharmonic operator with the Navier boundary conditions and its applications
}

\section{Futoshi Takahashi}

\begin{tabular}{|c|l|}
\hline Citation & OCAMI Preprint Series \\
\hline Issue Date & 2011 \\
\hline Type & Preprint \\
\hline Textversion & Author \\
\hline Rights & For personal use only. No other uses without permission. \\
\hline Relation & $\begin{array}{l}\text { This is the pre-peer reviewed version of the following article: Mathematische } \\
\text { Nachrichten, Vol.286, Issue2-3, p306-319, which has been published in final form } \\
\text { at https://doi.org/10.1002/mana.201100236 } . \text { This article may be used for } \\
\text { non-commercial purposes in accordance with Wiley Terms and Conditions for Use } \\
\text { of Self-Archived Versions. }\end{array}$ \\
\hline
\end{tabular}

From: Osaka City University Advanced Mathematical Institute http://www.sci.osaka-cu.ac.jp/OCAMI/publication/preprint/preprint.html 


\title{
Some identities of Green's function for the polyharmonic operator with the Navier boundary conditions and its applications
}

\author{
Futoshi Takahashi \\ Osaka City University \\ Department of Mathematics \& OCAMI \\ 3-3-138 Sugimoto, Sumiyoshi-ku \\ Osaka, 535-8585, Japan \\ Tel: $(+81)(0) 6-6605-2508$ \\ E-mail: futoshi@sci.osaka-cu.ac.jp
}

\begin{abstract}
We prove several integral identities for Green's function of the polyharmonic operator $(-\Delta)^{p}, p \in \mathbb{N}$ under the Navier boundary conditions. As an application, we prove the nondegeneracy of the critical point of the Robin function associated to the Green function on some symmetric domains.
\end{abstract}

\section{Introduction.}

Recently, many authors have been interested in the study of linear and nonlinear elliptic partial differential equations involving the higher-order differential operator, see for example, the recent book [5] and the reference therein.

2010 Mathematics Subject Classification: 35J08, 35J40.

Key words: Green's function, polyharmonic operator, Navier boundary condition. 
In the following, we fix $p \in \mathbb{N}$ and let $G=G(x, y)$ denote the Green function of $(-\Delta)^{p}$ under the Navier boundary condition:

$$
\left\{\begin{array}{l}
(-\Delta)^{p} G(\cdot, y)=\delta_{y} \quad \text { in } \Omega, \\
G(\cdot, y)=(-\Delta)^{j} G(\cdot, y)=0 \quad \text { on } \partial \Omega(j=1, \cdots p-1),
\end{array}\right.
$$

where $\Omega$ is a smooth bounded domain in $\mathbb{R}^{N}, N \geq 2 p$. Decompose $G$ as $G(x, y)=\Gamma(x, y)-H(x, y)$, where $\Gamma(x, y)$ is the fundamental solution of $(-\Delta)^{p}$ on $\mathbb{R}^{N}$ defined as

$$
\Gamma(x, y)= \begin{cases}C_{N, p}|x-y|^{2 p-N}, & N>2 p \\ C_{p} \log \frac{1}{|x-y|}, & N=2 p\end{cases}
$$

where

$$
\begin{aligned}
C_{N, p}=\frac{2 \Gamma\left(\frac{N}{2}-p\right)}{2^{2 p}(p-1) ! \Gamma\left(\frac{N}{2}\right) \sigma_{N}}, \quad N>2 p, \\
C_{p}=\frac{1}{\left\{2^{p-1}(p-1) !\right\}^{2} \sigma_{2 p}}, \quad N=2 p,
\end{aligned}
$$

and $\sigma_{N}=\frac{2 \pi^{N / 2}}{\Gamma(N / 2)}$ is the volume of the $(N-1)$ dimensional unit sphere in $\mathbb{R}^{N}$. $H=H(x, y) \in C^{\infty}(\Omega \times \Omega)$ is called the regular part of the Green function, and satisfies

$$
\left\{\begin{array}{l}
(-\Delta)^{p} H(\cdot, y)=0 \quad \text { in } \Omega \\
(-\Delta)^{j} H(\cdot, y)=(-\Delta)^{j} \Gamma(\cdot, y) \quad \text { on } \partial \Omega(j=0,1, \cdots p-1) .
\end{array}\right.
$$

Note that the coefficients in the expression of $\Gamma(x, y)$ are determined by the formula

$$
(-1)^{p} \int_{\partial B_{r}(0)} \frac{\partial \Delta^{p-1} \Gamma(x, 0)}{\partial \nu_{x}} d s_{x}=1
$$

here $\nu$ is the unit normal vector to $\partial B_{r}(0)$. Finally, let $R(y)=H(y, y)$ denote the Robin function of the Green function of $(-\Delta)^{p}$ with the Navier boundary condition.

In this paper, we prove the nondegeneracy of critical points of the Robin function on some symmetric domains. More precisely, let $\Omega$ be a smooth bounded domain in $\mathbb{R}^{N}, N \geq 2 p$, which is symmetric with respect to hyperplanes $\left\{x_{i}=0\right\}$ and convex in $x_{i}$-directions for $i=1, \cdots, N$. This kind of 
domains are sometimes called Gidas-Ni-Nirenberg domains (GNN domains for short) after the famous paper [6]. We will prove the Hessian matrix of the Robin function associated to the Green function of $(-\Delta)^{p}$ under the Navier boundary condition computed at the origin is diagonal and all diagonal elements are strictly positive. For the second order case $(p=1)$, this result was former proved by M. Grossi [7]. Basically our strategy of the proof is to follow his argument faithfully. However, in the course of the proof, we need to generalize some integral identities relating boundary integrations of the Green function to the Robin function, which, in turn, originate from the paper by Brezis and Peletier [1].

As for the second order case, it is known that the Robin function of $-\Delta$ with the Dirichlet boundary condition is strictly convex and has a unique nondegenerate critical point (global minimum point) on a bounded convex domain. This important fact was first proved by Caffarelli-Friedman [2] when $N=2$, and later extended to $N \geq 3$ by Cardaliaguet-Tahraoui [3]. Whether the same result holds true for the Robin function of $(-\Delta)^{p}$ with the Navier boundary condition is completely open. We hope the theorem mentioned above could shed light on this subject.

This paper is organized as follows. In $\S 2$, we recall some well-known facts on the Green function of $(-\Delta)^{p}$ under the Navier boundary conditions. $\S 3$ will be devoted to the proof of integral identities mentioned above, and we hope that this part would be useful in itself for some readers. In $\S 4$, we will prove the nondegeneracy of the critical point of the Robin function on GNN domains. $C$ will denote various constants from line to line until otherwise stated.

\section{Preliminaries.}

In this section, we recall some elementary facts that are useful later. First, we recall Green's 2nd identity

$$
\begin{aligned}
& \int_{\Omega}\left[\left(\Delta^{p} f\right) g-f\left(\Delta^{p} g\right)\right] d x \\
& =\sum_{k=1}^{p} \int_{\partial \Omega}\left[\left(\frac{\partial \Delta^{k-1} f}{\partial \nu_{x}}\right)\left(\Delta^{p-k} g\right)-\left(\Delta^{k-1} f\right)\left(\frac{\partial \Delta^{p-k} g}{\partial \nu_{x}}\right)\right] d s_{x},
\end{aligned}
$$

which holds for smooth $f, g$. 
In the following, we set $\bar{G}_{j}(x, y)=\left(-\Delta_{x}\right)^{j} G(x, y)$ for $j=0,1, \cdots, p-1$. Then $\bar{G}_{j}$ satisfies

$$
\left\{\begin{array}{l}
-\Delta_{x} \bar{G}_{j}=\bar{G}_{j+1} \quad \text { in } \Omega,(j=0,1, \cdots, p-2), \\
-\Delta_{x} \bar{G}_{p-1}=\delta_{y} \quad \text { in } \Omega, \\
\bar{G}_{j}>0 \text { in } \Omega,(j=0,1, \cdots, p-1), \\
\bar{G}_{j}=0 \quad \text { on } \partial \Omega,(j=0,1, \cdots, p-1) .
\end{array}\right.
$$

Note that $\bar{G}_{p-1}$ is the Green function of $-\Delta$ under the Dirichlet boundary condition. By using these symbols, the well-known Green's representation formula for the unique solution to the linear problem

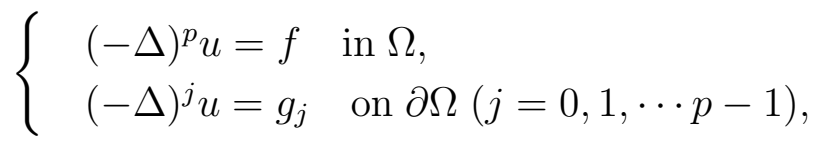

where $f$ and $g_{j}$ are smooth functions, can be written as follows:

$$
u(y)=\int_{\Omega} G(x, y) f(x) d x-\sum_{k=1}^{p} \int_{\partial \Omega}\left(\frac{\partial \bar{G}_{k-1}(x, y)}{\partial \nu_{x}}\right) g_{p-k}(x) d s_{x}
$$

for $y \in \Omega$.

Also we need a version of Pohozaev identity for the polyharmonic equation

$$
(-\Delta)^{p} u=f(u) \text { in } \Omega, \quad u \in C^{2 p}(\bar{\Omega})
$$

without boundary conditions.

Lemma 2.1 Assume $f \in C^{1}(\mathbb{R}, \mathbb{R})$. Then

$$
\begin{aligned}
& \int_{\Omega}\left[N F(u)-\left(\frac{N-2 p}{2}\right) u f(u)\right] d x=\int_{\partial \Omega}(x \cdot \nu)\left(F(u)-\frac{1}{2} u f(u)\right) d s_{x} \\
& +\frac{(-1)^{p-1}}{2} \sum_{k=1}^{p} \int_{\partial \Omega}\left[\left(\frac{\partial \Delta^{k-1} u}{\partial \nu}\right) \Delta^{p-k}(x \cdot \nabla u)-\left(\Delta^{k-1} u\right) \frac{\partial \Delta^{p-k}(x \cdot \nabla u)}{\partial \nu}\right] d s_{x}
\end{aligned}
$$

holds for a solution $u \in C^{2 p}(\bar{\Omega})$ to (2.4), where $F(u)=\int_{0}^{u} f(s) d s$. 
More general version is known, see [8], [9] and so on. We show a proof of the above lemma in order to make this paper self-contained.

Proof. By Green's 2nd identity (2.1) with $f=u, g=(x \cdot \nabla u)$, we have

$$
\begin{aligned}
& \int_{\Omega}\left[\left(\Delta^{p} u\right)(x \cdot \nabla u)-u \Delta^{p}(x \cdot \nabla u)\right] d x \\
& =\sum_{k=1}^{p} \int_{\partial \Omega}\left[\left(\frac{\partial \Delta^{k-1} u}{\partial \nu_{x}}\right) \Delta^{p-k}(x \cdot \nabla u)-\left(\Delta^{k-1} u\right)\left(\frac{\partial \Delta^{p-k}(x \cdot \nabla u)}{\partial \nu_{x}}\right)\right] d s_{x} \\
& =: \sum_{k=1}^{p} B_{k} .
\end{aligned}
$$

Note also that

$$
\Delta^{j}(x \cdot \nabla u)=2 j \Delta^{j} u+\left(x \cdot \nabla \Delta^{j} u\right), \quad(j=0,1,2, \cdots)
$$

which is easily shown by induction. Thus,

$$
\begin{aligned}
& \int_{\Omega} u \Delta^{p}(x \cdot \nabla u) d x=2 p \int_{\Omega} u \Delta^{p} u d x+\int_{\Omega} u\left(x \cdot \nabla \Delta^{p} u\right) d x \\
& =2 p(-1)^{p} \int_{\Omega} u f(u) d x+(-1)^{p} \int_{\Omega} u(x \cdot \nabla f(u)) d x \\
& =(-1)^{p}\left\{2 p \int_{\Omega} u f(u) d x+\int_{\Omega} \operatorname{div}(x(u f(u)-F(u))) d x+\int_{\Omega} N(F(u)-u f(u)) d x\right\} \\
& =(-1)^{p}\left\{\int_{\Omega}\{N F(u)-(N-2 p) u f(u)\} d x+\int_{\partial \Omega}(x \cdot \nu)(u f(u)-F(u)) d s_{x}\right\},
\end{aligned}
$$

where we have used (2.4) and the formula $u(x \cdot \nabla f(u))=\operatorname{div}(x(u f(u)-F(u)))+$ $N(F(u)-u f(u))$.

On the other hand,

$$
\begin{aligned}
& \int_{\Omega}\left(\Delta^{p} u\right)(x \cdot \nabla u) d x=(-1)^{p} \int_{\Omega} f(u)(x \cdot \nabla u) d x \\
& =(-1)^{p} \int_{\Omega}\{\operatorname{div}(x F(u))-N F(u)\} d x=(-1)^{p}\left\{\int_{\partial \Omega}(x \cdot \nu) F(u) d s_{x}-\int_{\Omega} N F(u) d x\right\} .
\end{aligned}
$$

Combining all together, we have

$$
\begin{aligned}
& (-1)^{p}\left\{\int_{\partial \Omega}(x \cdot \nu) F(u) d s_{x}-\int_{\Omega} N F(u) d x\right\}-\sum_{k=1}^{p} B_{k} \\
& =(-1)^{p}\left\{\int_{\Omega} N F(u)-(N-2 p) u f(u) d x+\int_{\partial \Omega}(x \cdot \nu)(u f(u)-F(u)) d s_{x}\right\},
\end{aligned}
$$


which implies the lemma.

\section{Integral identities for Green's function of $(-\Delta)^{p}$ with the Navier boundary conditions.}

In this section, we will prove some identities for the Green function of $(-\Delta)^{p}$ under the Navier boundary conditions. Part of these formulas were former proved by Brezis and Peletier [1] when $p=1, N>2$, Ren and Wei [10] when $p=1, N=2$, Chou and Geng [4] when $p=2, N>4$, and Takahashi [11] when $p=2, N=4$.

Theorem 3.1 For any $y \in \Omega$, we have

(1)

$$
\sum_{k=1}^{p} \int_{\partial \Omega}(x-y) \cdot \nu\left(\frac{\partial \bar{G}_{k-1}(x, y)}{\partial \nu_{x}}\right)\left(\frac{\partial \bar{G}_{p-k}(x, y)}{\partial \nu_{x}}\right) d s_{x}=(N-2 p) R(y)
$$

when $N>2 p$, and

$$
\sum_{k=1}^{p} \int_{\partial \Omega}(x-y) \cdot \nu\left(\frac{\partial \bar{G}_{k-1}(x, y)}{\partial \nu_{x}}\right)\left(\frac{\partial \bar{G}_{p-k}(x, y)}{\partial \nu_{x}}\right) d s_{x}=C_{p}
$$

when $N=2 p$, where $C_{p}$ is defined in (1.2).

(2)

$$
\sum_{k=1}^{p} \int_{\partial \Omega}\left(\frac{\partial \bar{G}_{k-1}(x, y)}{\partial \nu_{x}}\right)\left(\frac{\partial \bar{G}_{p-k}(x, y)}{\partial \nu_{x}}\right) \nu_{i}(x) d s_{x}=\frac{\partial R}{\partial y_{i}}(y)
$$

for $i=1, \cdots, N$ when $N \geq 2 p$.

(3)

$$
\begin{aligned}
& 2 \sum_{k=1}^{p} \int_{\partial \Omega}\left(\frac{\partial \bar{G}_{k-1}(x, y)}{\partial x_{i}}\right) \frac{\partial}{\partial y_{j}}\left(\frac{\partial \bar{G}_{p-k}(x, y)}{\partial \nu_{x}}\right) d s_{x}= \\
& 2 \sum_{k=1}^{p} \int_{\partial \Omega}\left(\frac{\partial \bar{G}_{p-k}(x, y)}{\partial x_{i}}\right) \frac{\partial}{\partial y_{j}}\left(\frac{\partial \bar{G}_{k-1}(x, y)}{\partial \nu_{x}}\right) d s_{x}=\frac{\partial^{2} R}{\partial y_{i} \partial y_{j}}(y)
\end{aligned}
$$

for $1 \leq i, j \leq N, N \geq 2 p$. 
Here $\nu=\nu(x)$ is the outer unit normal at $x \in \partial \Omega$.

Proof. First we prove (3.1). We may assume $y=0$ and choose $r>0$ small such that $B_{r}:=B_{r}(0) \subset \subset \Omega$. We apply the Pohozaev identity (2.5) in Lemma 2.1 to

$$
\left\{\begin{array}{l}
(-\Delta)^{p} G(\cdot, 0)=0 \quad \text { in } \Omega \backslash B_{r} \\
G(\cdot, 0)=(-\Delta)^{j} G(\cdot, 0)=0 \quad \text { on } \partial \Omega(j=1, \cdots p-1) .
\end{array}\right.
$$

Thus we obtain

$$
\begin{aligned}
& \sum_{k=1}^{p} \int_{\partial \Omega}\left(\frac{\partial \Delta^{k-1} G}{\partial \nu_{x}}\right) \Delta^{p-k}(x \cdot \nabla G) d s_{x} \\
& =\sum_{k=1}^{p} \int_{\partial B_{r}}\left[\left(\frac{\partial \Delta^{k-1} G}{\partial \nu_{x}}\right) \Delta^{p-k}(x \cdot \nabla G)-\left(\Delta^{k-1} G\right)\left(\frac{\partial \Delta^{p-k}(x \cdot \nabla G)}{\partial \nu_{x}}\right)\right] d s_{x},
\end{aligned}
$$

where $G=G(x, 0)$. Since $\Delta^{p-k}(x \cdot \nabla G)=2(p-k) \Delta^{p-k} G+\left(x \cdot \nabla \Delta^{p-k} G\right)=$ $\left(\frac{\partial \Delta^{p-k} G}{\partial \nu}\right)(x \cdot \nu)$ on $\partial \Omega$, we have

$$
\text { LHS of }(3.5)=\sum_{k=1}^{p} \int_{\partial \Omega}(x \cdot \nu)\left(\frac{\partial \Delta^{k-1} G(x, 0)}{\partial \nu}\right)\left(\frac{\partial \Delta^{p-k} G(x, 0)}{\partial \nu}\right) d s_{x} .
$$

On the other hand, inputting $G(x, 0)=\Gamma(x)-g(x)$ where $\Gamma(x)=\Gamma(x, 0)=$ $C_{N, p}|x|^{2 p-N}, g(x)=H(x, 0)$, we see

$$
\operatorname{RHS} \text { of }(3.5)=\sum_{k=1}^{p}\left(I_{1, k}-I_{2, k}-I_{3, k}+I_{4, k}\right),
$$

where

$$
\begin{aligned}
I_{1, k} & =\int_{\partial B_{r}}\left[\left(\frac{\partial \Delta^{k-1} \Gamma}{\partial \nu_{x}}\right) \Delta^{p-k}(x \cdot \nabla \Gamma)-\left(\Delta^{k-1} \Gamma\right)\left(\frac{\partial \Delta^{p-k}(x \cdot \nabla \Gamma)}{\partial \nu_{x}}\right)\right] d s_{x}, \\
I_{2, k}= & \int_{\partial B_{r}}\left[\left(\frac{\partial \Delta^{k-1} \Gamma}{\partial \nu_{x}}\right) \Delta^{p-k}(x \cdot \nabla g)-\left(\Delta^{k-1} \Gamma\right)\left(\frac{\partial \Delta^{p-k}(x \cdot \nabla g)}{\partial \nu_{x}}\right)\right] d s_{x}, \\
I_{3, k}= & \int_{\partial B_{r}}\left[\left(\frac{\partial \Delta^{k-1} g}{\partial \nu_{x}}\right) \Delta^{p-k}(x \cdot \nabla \Gamma)-\left(\Delta^{k-1} g\right)\left(\frac{\partial \Delta^{p-k}(x \cdot \nabla \Gamma)}{\partial \nu_{x}}\right)\right] d s_{x}, \\
I_{4, k}= & \int_{\partial B_{r}}\left[\left(\frac{\partial \Delta^{k-1} g}{\partial \nu_{x}}\right) \Delta^{p-k}(x \cdot \nabla g)-\left(\Delta^{k-1} g\right)\left(\frac{\partial \Delta^{p-k}(x \cdot \nabla g)}{\partial \nu_{x}}\right)\right] d s_{x} .
\end{aligned}
$$


First, we easily see that $I_{4, k}=o(1)$ as $r \rightarrow 0$ for any $k=1,2, \cdots, p$.

Next, we set $\Gamma(r)=C_{N, p} p^{2 p-N}$ for $r=|x|$. Then by induction, we have

$$
\begin{aligned}
\Delta^{l} \Gamma(r) & =C_{N, p} \prod_{i=0}^{l-1}(2 p-N-2 i) \prod_{j=1}^{l}(2 p-2 j) r^{2 p-N-2 l} \\
& =: A_{l} r^{2 p-N-2 l} \quad(l=0,1,2, \cdots,)
\end{aligned}
$$

where in this formula, we agree the convention that $\prod_{i=0}^{i=-1}(\cdots)=\prod_{j=1}^{j=0}(\cdots)=$ 1. Also, on $\partial B_{r}$, we see that

$$
\begin{aligned}
& \left(\frac{\partial \Delta^{l} \Gamma}{\partial \nu_{x}}\right)=\left(\Delta^{l} \Gamma\right)^{\prime}(r)=(2 p-N-2 l) A_{l} r^{2 p-N-2 l-1}, \\
& \left(x \cdot \nabla \Delta^{l} \Gamma\right)(x)=r\left(\Delta^{l} \Gamma\right)^{\prime}(r)=(2 p-N-2 l) A_{l} r^{2 p-N-2 l}, \\
& \Delta^{l}(x \cdot \nabla \Gamma)=2 l \Delta^{l} \Gamma+\left(x \cdot \nabla \Delta^{l} \Gamma\right)=(2 p-N) A_{l} r^{2 p-N-2 l}, \\
& \frac{\Delta^{l}(x \cdot \nabla \Gamma)}{\partial \nu_{x}}=\left(\Delta^{l}(x \cdot \nabla \Gamma)\right)^{\prime}(r)=(2 p-N)(2 p-N-2 l) A_{l} r^{2 p-N-2 l-1}
\end{aligned}
$$

holds for $l=0,1,2, \cdots$. Note that, by the formula (1.3), we have

$$
(-1)^{p}=\int_{\partial B_{r}(0)} \frac{\partial \Delta^{p-1} \Gamma(x, 0)}{\partial \nu_{x}} d s_{x}=(2-N) A_{p-1} \sigma_{N} .
$$

Therefore by (3.8) and (3.9), the integrand of $I_{1, k}$ is

$$
\begin{aligned}
& \left(\frac{\partial \Delta^{k-1} \Gamma}{\partial \nu_{x}}\right) \Delta^{p-k}(x \cdot \nabla \Gamma)-\left(\Delta^{k-1} \Gamma\right)\left(\frac{\partial \Delta^{p-k}(x \cdot \nabla \Gamma)}{\partial \nu_{x}}\right) \\
& =A_{k-1}(2 p-N-2 k+2) r^{2 p-N-2 k+1} \cdot(2 p-N) A_{p-k} r^{2 k-N} \\
& -A_{k-1} r^{2 p-N-2 k+2} \cdot A_{p-k}(2 p-N)(2 k-N) r^{2 k-N-1} \\
& =2(2 p-N) r^{2 p-2 N+1} A_{k-1} A_{p-k}(p-2 k+1),
\end{aligned}
$$

thus

$$
I_{1, k}=2 \sigma_{N}(2 p-N) r^{2 p-N} A_{k-1} A_{p-k}(p-2 k+1) .
$$

Since we easily check that

$$
\sum_{k=1}^{p} A_{k-1} A_{p-k}(p-2 k+1)=0
$$


for any $p \in \mathbb{N}$, we obtain that $\sum_{k=1}^{p} I_{1, k}=0$.

For $I_{2, k}$, by (3.9) we see that

$$
\begin{aligned}
\int_{\partial B_{r}}\left(\frac{\partial \Delta^{k-1} \Gamma}{\partial \nu_{x}}\right) \Delta^{p-k}(x \cdot \nabla g) d s_{x} & =C r^{2 p-2 k} \cdot r^{1-N} \int_{\partial B_{r}} \text { (smooth function) } d s_{x} \\
& =o(1) \quad \text { as } r \rightarrow 0, k \in\{1,2, \cdots, p-1\} .
\end{aligned}
$$

Also when $k=p$,

$$
\int_{\partial B_{r}}\left(\frac{\partial \Delta^{p-1} \Gamma}{\partial \nu_{x}}\right)(x \cdot \nabla g) d s_{x}=C r^{1-N} \int_{\partial B_{r}} r\left(\frac{\partial g}{\partial \nu}\right) d s_{x} \rightarrow 0 \quad \text { as } r \rightarrow 0 .
$$

Similarly, we have

$$
\begin{aligned}
\int_{\partial B_{r}}\left(\Delta^{k-1} \Gamma\right)\left(\frac{\partial \Delta^{p-k}(x \cdot \nabla g)}{\partial \nu_{x}}\right) d s_{x} & =C r^{2 p-2 k+1} \cdot r^{1-N} \int_{\partial B_{r}} \text { (smooth function) } d s_{x} \\
& =o(1) \quad \text { as } r \rightarrow 0, k \in\{1,2, \cdots, p\} .
\end{aligned}
$$

Combining these, we obtain $I_{2, k}=o(1)$ as $r \rightarrow 0$ for all $k=1, \cdots, p$.

For $I_{3, k}$, we see by $(3.9)$,

$$
\begin{aligned}
\int_{\partial B_{r}}\left(\frac{\partial \Delta^{k-1} g}{\partial \nu_{x}}\right) \Delta^{p-k}(x \cdot \nabla \Gamma) d s_{x} & =C r^{2 k-1} \cdot r^{1-N} \int_{\partial B_{r}}(\text { smooth function }) d s_{x} \\
& =o(1) \quad \text { as } r \rightarrow 0, k \in\{1,2, \cdots, p\} . \\
\int_{\partial B_{r}}\left(\Delta^{k-1} g\right)\left(\frac{\partial \Delta^{p-k}(x \cdot \nabla \Gamma)}{\partial \nu_{x}}\right) d s_{x} & =C r^{2 k-2} \cdot r^{1-N} \int_{\partial B_{r}}\left(\Delta^{k-1} g\right) d s_{x} \\
& =o(1) \quad \text { as } r \rightarrow 0, k \in\{2,3, \cdots, p\} .
\end{aligned}
$$

On the other hand, when $k=1$,

$$
\begin{aligned}
\int_{\partial B_{r}} g(x)\left(\frac{\partial \Delta^{p-1}(x \cdot \nabla \Gamma)}{\partial \nu_{x}}\right) d s_{x} & =(2 p-N)(2-N) A_{p-1} \cdot r^{1-N} \int_{\partial B_{r}} g(x) d s_{x} \\
& \rightarrow(2 p-N)(2-N) A_{p-1} \cdot \sigma_{N} g(0) \\
& =(2 p-N)(-1)^{p} g(0)
\end{aligned}
$$

as $r \rightarrow 0$, here we have used (3.10). Combining these, we obtain that

$$
-\sum_{k=1}^{p} I_{k}^{3}=\int_{\partial B_{r}} g(x)\left(\frac{\partial \Delta^{p-1}(x \cdot \nabla \Gamma)}{\partial \nu_{x}}\right) d s_{x}+o(1)=(N-2 p)(-1)^{p-1} g(0)+o(1)
$$


as $r \rightarrow 0$.

Returning to (3.6), (3.7) with these estimates, we obtain

$\sum_{k=1}^{p} \int_{\partial \Omega}(x \cdot \nu)\left(\frac{\partial \Delta^{k-1} G(x, 0)}{\partial \nu}\right)\left(\frac{\partial \Delta^{p-k} G(x, 0)}{\partial \nu}\right) d s_{x}=(-1)^{p-1}(N-2 p) g(0)$, which leads to (3.1) when $N>2 p$.

Next, we prove (3.2) when $N=2 p$. We treat the case when $p \geq 2$ only, since the formula for $p=1(N=2)$ :

$$
\int_{\partial \Omega}(x-y) \cdot \nu(x)\left(\frac{\partial G(x, y)}{\partial \nu_{x}}\right)^{2} d s_{x}=\frac{1}{2 \pi}\left(=C_{1}\right)
$$

holds for any $y \in \Omega$ similarly.

Now, $\Gamma(r)=-C_{p} \log r$ where $C_{p}$ is defined as (1.2), therefore, we have on $\partial B_{r}$

$$
\begin{aligned}
& \Delta^{l} \Gamma(r)=2^{l-1}(l-1) ! C_{p} \prod_{i=1}^{l}(2 i-2 p) r^{-2 l}=: B_{l} r^{-2 l}, \\
& \left(\frac{\partial^{l} \Gamma}{\partial \nu_{x}}\right)=\left(\Delta^{l} \Gamma\right)^{\prime}(r)=(-2 l) B_{l} r^{-2 l-1}, \\
& \left(x \cdot \nabla \Delta^{l} \Gamma\right)(x)=r\left(\Delta^{l} \Gamma\right)^{\prime}(r)=(-2 l) B_{l} r^{-2 l}, \\
& \Delta^{l}(x \cdot \nabla \Gamma)=2 l \Delta^{l} \Gamma+\left(x \cdot \Delta^{l} \Gamma\right)=2 l B_{l} r^{-2 l}+(-2 l) B_{l} r^{-2 l}=0, \\
& \frac{\Delta^{l}(x \cdot \nabla \Gamma)}{\partial \nu_{x}}=\left(\Delta^{l}(x \cdot \nabla \Gamma)\right)^{\prime}(r)=0
\end{aligned}
$$

for $l=1,2, \cdots, p$. Note that $\Delta^{p} \Gamma(r)=0$. Just as before, we have (3.5), (3.6), (3.7), and $I_{4, k}=o(1)$ as $r \rightarrow 0$ for $k=1, \cdots, p$.

For $I_{1, k}$, since $\Delta^{p-k}(x \cdot \nabla \Gamma)=\frac{\Delta^{p-k}(x \cdot \nabla \Gamma)}{\partial \nu_{x}}=0$ for $k \neq p$, we have $I_{1, k}=0$ for $k \in\{1,2, \cdots, p-1\}$. On the other hand, since

we see

$$
\begin{aligned}
& x \cdot \nabla \Gamma=r(\Gamma(r))^{\prime}=-C_{p}, \\
& \frac{\partial \Delta^{p-1} \Gamma}{\partial \nu}=\left(\Delta^{p-1} \Gamma\right)^{\prime}(r)=B_{p-1}(2-2 p) r^{1-2 p}, \quad(p \geq 2),
\end{aligned}
$$

$$
\begin{aligned}
I_{1, p} & =\int_{\partial B_{r}}\left[\left(\frac{\partial \Delta^{p-1} \Gamma}{\partial \nu_{x}}\right)(x \cdot \nabla \Gamma)-\left(\Delta^{p-1} \Gamma\right)\left(\frac{\partial(x \cdot \nabla \Gamma)}{\partial \nu_{x}}\right)\right] d s_{x} \\
& =\int_{\partial B_{r}}\left(-C_{p}\right) B_{p-1}(2-2 p) r^{1-2 p} d s_{x}=B_{p-1} C_{p} 2(p-1) \sigma_{2 p} .
\end{aligned}
$$


Again we check that $2(p-1) B_{p-1} \sigma_{2 p}=(-1)^{p-1}$ by $(1.3)$. Thus we have

$$
I_{1, k}= \begin{cases}0, & k \in\{1,2, \cdots, p-1\}, \\ (-1)^{p-1} C_{p}, & k=p .\end{cases}
$$

Also, since $\Delta^{k-1} \Gamma(r)=B_{p-1} r^{-2(k-1)}, \frac{\partial \Delta^{k-1} \Gamma}{\partial \nu}(r)=B_{k-1}(2-2 k) r^{1-2 k}$ for $k \in\{2,3, \cdots, p\}$, we easily check that $I_{2, k}=o(1)$ as $r \rightarrow 0$ just as before for $k \in\{2,3, \cdots, p\}$. For $I_{2,1}$, we see

$$
\begin{aligned}
I_{2,1} & =\int_{\partial B_{r}}\left[\left(\frac{\partial \Gamma}{\partial \nu_{x}}\right) \Delta^{p-1}(x \cdot \nabla g)-\Gamma\left(\frac{\partial \Delta^{p-1}(x \cdot \nabla g)}{\partial \nu_{x}}\right)\right] d s_{x} \\
& =\int_{\partial B_{r}}\left(-C_{p}\right)\left(\frac{1}{r}\right) \Delta^{p-1}(x \cdot \nabla g) d s_{x}+C_{p} \int_{\partial B_{r}} \log r\left(\frac{\partial \Delta^{p-1}(x \cdot \nabla g)}{\partial \nu_{x}}\right) d s_{x} \\
& =\left(r^{2 p-2}+r^{2 p-1} \log r\right) \int_{S^{2 p-1}} \text { (smooth function) } d \omega \\
& =o(1)
\end{aligned}
$$

as $r \rightarrow 0$ when $p \geq 2$. Thus we have $I_{2, k}=o(1)$ for all $k \in\{1,2,3, \cdots, p\}$.

For $I_{3, k}$, since $\Delta^{p-k}(x \cdot \Gamma)=0$ for $k \neq p$, we see $I_{3, k}=0$ for $k \neq p$. Also, since $x \cdot \nabla \Gamma=-C_{p}$, we see

$$
\begin{aligned}
I_{3, p} & =\int_{\partial B_{r}}\left[\left(\frac{\partial \Delta^{p-1} g}{\partial \nu_{x}}\right)(x \cdot \nabla \Gamma)-\left(\Delta^{p-1} g\right)\left(\frac{\partial(x \cdot \nabla \Gamma)}{\partial \nu_{x}}\right)\right] d s_{x} \\
& =\left(-C_{p}\right) \int_{\partial B_{r}}\left(\frac{\partial \Delta^{p-1} g}{\partial \nu_{x}}\right) d s_{x}=o(1)
\end{aligned}
$$

as $r \rightarrow 0$.

Returning to (3.6), (3.7), we obtain

$$
\sum_{k=1}^{p} \int_{\partial \Omega}(x \cdot \nu)\left(\frac{\partial \Delta^{k-1} G(x, 0)}{\partial \nu}\right)\left(\frac{\partial \Delta^{p-k} G(x, 0)}{\partial \nu}\right) d s_{x}=(-1)^{p-1} C_{p},
$$

which ends the proof of (3.2).

To prove (3.3), we apply Green's 2nd identity (2.1) for $f=G=G(x, 0)$, $g=G_{x_{i}}$ on $\Omega \backslash B_{r}(0)$. Since $\Delta^{p} G=\left(\Delta^{p} G\right)_{x_{i}} \equiv 0$ on $\Omega \backslash B_{r}(0)$, we get

$$
0=\sum_{k=1}^{p} \int_{\partial\left(\Omega \backslash B_{r}\right)}\left[\left(\frac{\partial \Delta^{k-1} G}{\partial \nu_{x}}\right)\left(\Delta^{p-k} G\right)_{x_{i}}-\left(\Delta^{k-1} G\right)\left(\frac{\partial\left(\Delta^{p-k} G\right)_{x_{i}}}{\partial \nu_{x}}\right)\right] d s_{x}
$$


which leads to

$$
\begin{aligned}
& \sum_{k=1}^{p} \int_{\partial \Omega}\left(\frac{\partial \Delta^{k-1} G}{\partial \nu_{x}}\right)\left(\Delta^{p-k} G\right)_{x_{i}} d s_{x} \\
& =\sum_{k=1}^{p} \int_{\partial B_{r}}\left[\left(\frac{\partial \Delta^{k-1} G}{\partial \nu_{x}}\right)\left(\Delta^{p-k} G\right)_{x_{i}}-\left(\Delta^{k-1} G\right)\left(\frac{\partial\left(\Delta^{p-k} G\right)_{x_{i}}}{\partial \nu_{x}}\right)\right] d s_{x} .
\end{aligned}
$$

Since $\left(\Delta^{p-k} G\right)_{x_{i}}=\left(\frac{\partial \Delta^{p-k} G}{\partial \nu_{x}}\right) \nu_{i}(x)$ on $\partial \Omega$, we see

$$
\operatorname{LHS} \text { of }(3.12)=\sum_{k=1}^{p} \int_{\partial \Omega}\left(\frac{\partial \Delta^{k-1} G(x, 0)}{\partial \nu_{x}}\right)\left(\frac{\partial \Delta^{p-k} G(x, 0)}{\partial \nu_{x}}\right) \nu_{i}(x) d s_{x} \text {. }
$$

On the other hand, inputting $G(x, 0)=\Gamma(x)-g(x), g(x)=H(x, 0)$ as before, we obtain

$$
\operatorname{RHS} \text { of }(3.5)=\sum_{k=1}^{p}\left(J_{1, k}-J_{2, k}-J_{3, k}+J_{4, k}\right)
$$

where

$$
\begin{aligned}
& J_{1, k}=\int_{\partial B_{r}}\left[\left(\frac{\partial \Delta^{k-1} \Gamma}{\partial \nu_{x}}\right)\left(\Delta^{p-k} \Gamma\right)_{x_{i}}-\left(\Delta^{k-1} \Gamma\right)\left(\frac{\partial\left(\Delta^{p-k} \Gamma\right)_{x_{i}}}{\partial \nu_{x}}\right)\right] d s_{x} \\
& J_{2, k}=\int_{\partial B_{r}}\left[\left(\frac{\partial \Delta^{k-1} \Gamma}{\partial \nu_{x}}\right)\left(\Delta^{p-k} g\right)_{x_{i}}-\left(\Delta^{k-1} \Gamma\right)\left(\frac{\partial\left(\Delta^{p-k} g\right)_{x_{i}}}{\partial \nu_{x}}\right)\right] d s_{x} \\
& J_{3, k}=\int_{\partial B_{r}}\left[\left(\frac{\partial \Delta^{k-1} g}{\partial \nu_{x}}\right)\left(\Delta^{p-k} \Gamma\right)_{x_{i}}-\left(\Delta^{k-1} g\right)\left(\frac{\partial\left(\Delta^{p-k} \Gamma\right)_{x_{i}}}{\partial \nu_{x}}\right)\right] d s_{x} \\
& J_{4, k}=\int_{\partial B_{r}}\left[\left(\frac{\partial \Delta^{k-1} g}{\partial \nu_{x}}\right)\left(\Delta^{p-k} g\right)_{x_{i}}-\left(\Delta^{k-1} g\right)\left(\frac{\partial\left(\Delta^{p-k} g\right)_{x_{i}}}{\partial \nu_{x}}\right)\right] d s_{x}
\end{aligned}
$$

Again, we see that $J_{4, k}=o(1)$ as $r \rightarrow 0$ for any $k=1,2, \cdots, p, N \geq 2 p$. 
Now, we treat the case $N>2 p$. In this case, since $\Delta^{l} \Gamma=A_{l} r^{2 p-N-2 l}$ by (3.8), we have

$$
\begin{aligned}
& \left(\Delta^{l} \Gamma\right)_{x_{i}}=A_{l}(2 p-N-2 l) r^{2 p-N-2 l-1} \nu_{i}(x) \\
& \frac{\partial\left(\Delta^{l} \Gamma\right)_{x_{i}}}{\partial \nu_{x}}=\frac{x}{r} \cdot \nabla\left(\Delta^{l} \Gamma\right)_{x_{i}} \\
& =\frac{x}{r} \cdot A_{l}(2 p-N-2 l)\left\{(2 p-N-2 l-2) r^{2 p-N-2 l-3} x_{i} \frac{x}{r}+r^{2 p-N-2 l-2} e_{i}\right\} \\
& =A_{l}(2 p-N-2 l)(2 p-N-2 l-1) r^{2 p-N-2 l-2} \nu_{i}(x)
\end{aligned}
$$

for $l=0,1,2, \cdots$ on $\partial B_{r}$, here $e_{i}=\nabla x_{i}$ and we have used $\nu_{i}(x)=\frac{x_{i}}{r}$ on $\partial B_{r}$ for $i=1,2, \cdots, N$. By (3.8) and (3.15), we have

$$
\begin{aligned}
J_{1, k} & =\int_{\partial B_{r}}\left[\left(\frac{\partial \Delta^{k-1} \Gamma}{\partial \nu_{x}}\right)\left(\Delta^{p-k} \Gamma\right)_{x_{i}}-\Delta^{k-1} \Gamma\left(\frac{\partial\left(\Delta^{p-k} \Gamma\right)_{x_{i}}}{\partial \nu_{x}}\right)\right] d s_{x} \\
& =C \int_{\partial B_{r}} r^{2 p-N-2 k+1} \cdot r^{2 k-N-1} \nu_{i}(x) d s_{x}-C^{\prime} \int_{\partial B_{r}} r^{2 p-N-2 k+2} \cdot r^{2 k-N-2} \nu_{i}(x) d s_{x} \\
& =C^{\prime \prime} r^{2(p-N)} \int_{\partial B_{r}} \nu_{i}(x) d s_{x}=0, \quad k \in\{1,2 \cdots, p\},
\end{aligned}
$$

here we have used $\int_{\partial B_{r}} \nu_{i}(x) d s_{x}=0$.

As for the estimate of $J_{2, k}$, as in the proof of (3.1), we see

$$
\begin{aligned}
\int_{\partial B_{r}}\left(\frac{\partial \Delta^{k-1} \Gamma}{\partial \nu_{x}}\right)\left(\Delta^{p-k} g\right)_{x_{i}} d s_{x} & =C r^{2(p-k)} \cdot r^{1-N} \int_{\partial B_{r}} \text { (smooth function) } d s_{x} \\
& =o(1), \quad(k=1,2, \cdots, p-1) \\
\int_{\partial B_{r}}\left(\frac{\partial \Delta^{p-1} \Gamma}{\partial \nu_{x}}\right) g_{x_{i}} d s_{x} & =A_{p-1}(2-N) r^{1-N} \int_{\partial B_{r}} g_{x_{i}} d s_{x} \\
& \rightarrow A_{p-1}(2-N) \sigma_{N} g_{x_{i}}(0)=(-1)^{p} g_{x_{i}}(0), \\
\int_{\partial B_{r}}\left(\Delta^{k-1} \Gamma\right)\left(\frac{\partial\left(\Delta^{p-k} g\right)_{x_{i}}}{\partial \nu_{x}}\right) d s_{x} & =C r^{2 p-2 k+1} \cdot r^{1-N} \int_{\partial B_{r}}(\text { smooth function }) d s_{x} \\
& =o(1) \quad(k=1,2, \cdots, p) .
\end{aligned}
$$

Thus we have

$$
J_{2, k}= \begin{cases}o(1), & k \in\{1,2, \cdots, p-1\}, \\ (-1)^{p} g_{x_{i}}(0)+o(1), & k=p\end{cases}
$$


as $r \rightarrow 0$.

As for the estimate of $J_{3, k}$, we see

$$
\begin{aligned}
\int_{\partial B_{r}}\left(\frac{\partial \Delta^{k-1} g}{\partial \nu_{x}}\right)\left(\Delta^{p-k} \Gamma\right)_{x_{i}} d s_{x} & =C r^{2 k-2} \cdot r^{1-N} \int_{\partial B_{r}}\left(\frac{\partial \Delta^{k-1} g}{\partial \nu_{x}}\right) \nu_{i}(x) d s_{x} \\
& =o(1), \quad(k=2,3 \cdots, p), \\
\int_{\partial B_{r}}\left(\Delta^{k-1} g\right)\left(\frac{\partial\left(\Delta^{p-k} \Gamma\right)_{x_{i}}}{\partial \nu_{x}}\right) d s_{x} & =C r^{2 k-3} \cdot r^{1-N} \int_{\partial B_{r}}\left(\Delta^{k-1} g\right) \nu_{i}(x) d s_{x} \\
& =o(1), \quad(k=2,3 \cdots, p),
\end{aligned}
$$

thus we have $J_{3, k}=o(1)$ for $k \in\{2,3, \cdots, p\}$. Now, we estimate $J_{3,1}$. For smooth $g$, we have

$$
\begin{aligned}
& \int_{\partial B_{r}}\left(\frac{\partial g}{\partial \nu_{x}}\right) \nu_{i}(x) d s_{x}=\int_{\partial B_{r}}\left(\sum_{j=1}^{N} \frac{\partial g}{\partial x_{j}} \nu_{j}(x)\right) \nu_{i}(x) d s_{x} \\
& =\sum_{j=1}^{N} \int_{\partial B_{r}}\left(\frac{\partial g}{\partial x_{j}}(x)-\frac{\partial g}{\partial x_{j}}(0)\right) \nu_{j} \nu_{i} d s_{x}+\sum_{j=1}^{N} \int_{\partial B_{r}} \frac{\partial g}{\partial x_{j}}(0) \nu_{j} \nu_{i} d s_{x} \\
& =O(r) \cdot O\left(r^{N-1}\right)+\frac{\partial g}{\partial x_{i}}(0) \frac{\sigma_{N}}{N} r^{N-1}
\end{aligned}
$$

where we have used

$$
\int_{\partial B_{r}} \nu_{i} \nu_{j} d s_{x}= \begin{cases}0, & (i \neq j), \\ \frac{\sigma_{N}}{N} r^{N-1}, & (i=j) .\end{cases}
$$

Thus we obtain

$$
\begin{aligned}
\int_{\partial B_{r}}\left(\frac{\partial g}{\partial \nu_{x}}\right)\left(\Delta^{p-1} \Gamma\right)_{x_{i}} d s_{x} & =A_{p-1}(2-N) r^{1-N} \int_{\partial B_{r}}\left(\frac{\partial g}{\partial \nu_{x}}\right) \nu_{i}(x) d s_{x} \\
& \rightarrow A_{p-1}(2-N) \frac{\sigma_{N}}{N} g_{x_{i}}(0), \quad \text { as } r \rightarrow 0 .
\end{aligned}
$$

Also by Taylor expansion, we have

$$
\begin{aligned}
& \int_{\partial B_{r}} g\left(\frac{\partial\left(\Delta^{p-1} \Gamma\right)_{x_{i}}}{\partial \nu_{x}}\right) d s_{x} \\
& =A_{p-1}(2-N)(1-N) r^{-N} \int_{\partial B_{r}}\left(g(0)+\nabla g(0) \cdot x+O\left(|x|^{2}\right)\right) \nu_{i}(x) d s_{x} .
\end{aligned}
$$


Since

$$
\begin{aligned}
& r^{-N} \int_{\partial B_{r}} g(0) \nu_{i}(x) d s_{x}=0 \\
& r^{-N} \int_{\partial B_{r}} O\left(|x|^{2}\right) \nu_{i}(x) d s_{x}=O\left(r^{2-N}\right) \times O\left(r^{1-N}\right)=O(r) \rightarrow 0 \\
& r^{-N} \int_{\partial B_{r}}(\nabla g(0) \cdot x) \nu_{i}(x) d s_{x}=r^{1-N} \sum_{j=1}^{N} \frac{\partial g}{\partial x_{j}}(0) \int_{\partial B_{r}} \nu_{j} \nu_{i} d s_{x}=\frac{\sigma_{N}}{N} \frac{\partial g}{\partial x_{i}}(0),
\end{aligned}
$$

we obtain that

$$
\int_{\partial B_{r}} g\left(\frac{\partial\left(\Delta^{p-1} \Gamma\right)_{x_{i}}}{\partial \nu_{x}}\right) d s_{x} \rightarrow A_{p-1}(2-N)(1-N) \frac{\sigma_{N}}{N} \frac{\partial g}{\partial x_{i}}(0)
$$

as $r \rightarrow 0$. Thus, by (3.10), we have

$$
\begin{aligned}
J_{3,1} & \rightarrow A_{p-1}(2-N) \frac{\sigma_{N}}{N} g_{x_{i}}(0)-A_{p-1}(2-N)(1-N) \frac{\sigma_{N}}{N} g_{x_{i}}(0) \\
& =A_{p-1}(2-N) \sigma_{N} g_{x_{i}}(0)=(-1)^{p} g_{x_{i}}(0) .
\end{aligned}
$$

Returning to (3.13), (3.14) with the above estimates, we have

$$
\begin{aligned}
& \sum_{k=1}^{p} \int_{\partial \Omega}\left(\frac{\partial \Delta^{k-1} G(x, 0)}{\partial \nu_{x}}\right)\left(\frac{\partial \Delta^{p-k} G(x, 0)}{\partial \nu_{x}}\right)(x, y) \nu_{i}(x) d s_{x} \\
& =0-J_{2, p}-J_{3,1}+o(1)=-(-1)^{p} g_{x_{i}}(0)-(-1)^{p} g_{x_{i}}(0)+o(1) \\
& =(-1)^{p-1} R_{x_{i}}(0)+o(1)
\end{aligned}
$$

where we have used that $g_{x_{i}}(0)=\left.\frac{\partial}{\partial x_{i}} H(x, 0)\right|_{x=0}=\frac{1}{2} R_{x_{i}}(0)$. Letting $r \rightarrow 0$, we have (3.3) when $N>2 p$.

Next, we prove (3.3) when $N=2 p$. The argument is almost the same as before, so we should be brief. Again we only treat the case $p \geq 2$, since the formula was proved in [10] when $p=1$. Recall $\Gamma(r)=-C_{p} \log r$ and $\Delta^{l} \Gamma=B_{l} r^{-2 l}$ for $l=1,2, \cdots$, on $\partial B_{r}$, here $B_{l}$ is defined in (3.11). Note that $B_{l}=0$ for $l \geq p$. Thus if we put

$$
\tilde{B}_{l}:=(-2 l) B_{l}=-C_{p} 2^{l} l ! \prod_{i=1}^{l}(2 i-2 p)
$$


and we agree the convention that $\tilde{B}_{0}=-C_{p}$, we have

$$
\begin{aligned}
& \left(\Delta^{l} \Gamma\right)_{x_{i}}=\tilde{B}_{l} r^{-2 l-1} \nu_{i}(x), \\
& \frac{\partial\left(\Delta^{l} \Gamma\right)_{x_{i}}}{\partial \nu_{x}}=-(2 l+1) \tilde{B}_{l} r^{-2 l-2} \nu_{i}(x)
\end{aligned}
$$

for $l=0,1,2, \cdots$, on $\partial B_{r}$. By using (3.11) and (3.16), we obtain, as before,

$$
\begin{aligned}
& J_{1, k}=0, \quad k \in\{1,2, \cdots, p\}, \\
& J_{2, k}=\left\{\begin{array}{l}
o(1), \quad k \in\{1,2, \cdots, p-1\} \\
\tilde{B}_{p-1} \sigma_{N} g_{x_{i}}(0)+o(1), \quad k=p
\end{array}\right. \\
& J_{3, k}=o(1), \quad k \in\{2,3, \cdots, p\}, \\
& J_{3,1}=\tilde{B}_{p-1} \frac{\sigma_{N}}{N} g_{x_{i}}(0)-\left\{-(2 p-1) \tilde{B}_{p-1} \frac{\sigma_{N}}{N} g_{x_{i}}(0)\right\}+o(1) \\
& =\tilde{B}_{p-1} \sigma_{N} g_{x_{i}}(0)+o(1)
\end{aligned}
$$

as $r \rightarrow 0$, where $\tilde{B}_{p-1} \sigma_{N}=(-1)^{p}$ by (1.3). Thus returning to (3.13), (3.14), we obtain (3.3) when $N=2 p$.

Finally, we prove (3.4). Differentiating (3.3) with respect to $y_{j}$, we obtain

$$
\begin{aligned}
& \frac{\partial^{2} R}{\partial y_{i} \partial y_{j}}(y) \\
& =\sum_{k=1}^{p} \int_{\partial \Omega}\left[\left\{\frac{\partial}{\partial y_{j}}\left(\frac{\partial \bar{G}_{k-1}}{\partial \nu_{x}}\right)\right\}\left(\frac{\partial \bar{G}_{p-k}}{\partial \nu_{x}}\right) \nu_{i}(x)+\left(\frac{\partial \bar{G}_{k-1}}{\partial \nu_{x}}\right) \nu_{i}(x) \frac{\partial}{\partial y_{j}}\left(\frac{\partial \bar{G}_{p-k}}{\partial \nu_{x}}\right)\right] d s_{x} .
\end{aligned}
$$

Note that $\left(\frac{\partial \bar{G}_{j}}{\partial \nu_{x}}(x, y)\right) \nu_{i}(x)=\frac{\partial \bar{G}_{j}}{\partial x_{i}}(x, y)$ for any $j=0,1, \cdots, p-1$ on $\partial \Omega$ since $\bar{G}_{j}=0$ on $\partial \Omega$. Thus we have

$$
\begin{aligned}
\frac{\partial^{2} R}{\partial y_{i} \partial y_{j}}(y) & =\sum_{k=1}^{p} \int_{\partial \Omega}\left[\left\{\frac{\partial}{\partial y_{j}}\left(\frac{\partial \bar{G}_{k-1}}{\partial \nu_{x}}\right)\right\}\left(\frac{\partial \bar{G}_{p-k}}{\partial x_{i}}\right)+\left(\frac{\partial \bar{G}_{k-1}}{\partial x_{i}}\right) \frac{\partial}{\partial y_{j}}\left(\frac{\partial \bar{G}_{p-k}}{\partial \nu_{x}}\right)\right] d s_{x} \\
& =2 \sum_{k=1}^{p} \int_{\partial \Omega}\left(\frac{\partial \bar{G}_{p-k}(x, y)}{\partial x_{i}}\right) \frac{\partial}{\partial y_{j}}\left(\frac{\partial \bar{G}_{k-1}(x, y)}{\partial \nu_{x}}\right) d s_{x} \\
& =2 \sum_{k=1}^{p} \int_{\partial \Omega}\left(\frac{\partial \bar{G}_{k-1}(x, y)}{\partial x_{i}}\right) \frac{\partial}{\partial y_{j}}\left(\frac{\partial \bar{G}_{p-k}(x, y)}{\partial \nu_{x}}\right) d s_{x}
\end{aligned}
$$




\section{Nondegeneracy of critical points of the Robin function on symmetric domains.}

In this section, we prove the nondegeneracy of the critical point of the Robin function associated to the Green function of $(-\Delta)^{p}$ with the Navier boundary conditions on some symmetric domains. Let $\Omega \subset \mathbb{R}^{N},(N \geq 2 p)$ be a smooth bounded domain. We call $\Omega$ a GNN domain, if the followings hold.

(H1) $\Omega$ is symmetric with respect to hyperplanes $\left\{x_{i}=0\right\}(i=1, \cdots, N)$.

(H2) $\Omega$ is convex with respect to $x_{i}$-directions $(i=1, \cdots, N)$.

See [6]. Note that a GNN domain need not be convex.

In this section we prove the following theorem, which extends the result obtained by Grossi [7] when $p=1$ to the general case $p \in \mathbb{N}$.

Theorem 4.1 Let $\Omega \subset \mathbb{R}^{N},(N \geq 2 p)$ be a smooth bounded domain with (H1), (H2). Let $R=R(y)$ be the Robin function of $(-\Delta)^{p}$ under the Navier boundary condition. Then we have

$$
\nabla_{y} R(0)=0, \quad \frac{\partial^{2} R}{\partial y_{i} \partial y_{j}}(0)=\left\{\begin{array}{l}
0 \quad(i \neq j) \\
a_{i}>0 \quad(i=j)
\end{array}\right.
$$

holds true.

We proceed as in [7]. First, we prepare some lemmas. In the following, let us denote $x=\left(x_{1}, x^{\prime}\right) \in \Omega, x^{\prime}=\left(x_{2}, \cdots, x_{N}\right) \in \mathbb{R}^{N-1}$.

Lemma 4.2 Assume $\Omega$ is symmetric with respect to the hyperplane $\left\{x_{1}=0\right\}$ and set $\Omega_{0}=\Omega \cap\left\{x_{1}=0\right\}$. Then for any $y_{0} \in \Omega_{0}$, we have

$$
\bar{G}_{k}\left(\left(x_{1}, x^{\prime}\right), y_{0}\right)=\bar{G}_{k}\left(\left(-x_{1}, x^{\prime}\right), y_{0}\right), \quad \forall k=1,2, \cdots, p-1 .
$$

Proof. By Lemma 2.1 of [7], we know that $\bar{G}_{p-1}\left(x, y_{0}\right)$ is even with respect to $x_{1}$-variable. Let us fix any $\phi \in C_{0}^{\infty}(\Omega)$. By $(2.2),-\Delta \bar{G}_{p-2}\left(x, y_{0}\right)=$ $\bar{G}_{p-1}\left(x, y_{0}\right)$ for $x \in \Omega$. Since $\Omega$ is symmetric with respect to the plane $\left\{x_{1}=\right.$ $0\}$ and $y_{0} \in \Omega_{0}$, we also have $-\Delta \bar{G}_{p-1}\left(\left(-x_{1}, x^{\prime}\right), y_{0}\right)=\bar{G}_{p-1}\left(\left(-x_{1}, x^{\prime}\right), y_{0}\right)$, thus $-\Delta \bar{G}_{p-1}\left(\left(-x_{1}, x^{\prime}\right), y_{0}\right)=\bar{G}_{p-1}\left(x, y_{0}\right)$ for $x \in \Omega$, since $\bar{G}_{p-1}\left(x, y_{0}\right)$ is even with respect to $x_{1}$. 
Multiplying $\phi$ to these equations, we get

$$
\int_{\Omega}\left\{\nabla \bar{G}_{p-2}\left(\left(x_{1}, x^{\prime}\right), y_{0}\right)-\nabla \bar{G}_{p-2}\left(\left(-x_{1}, x^{\prime}\right), y_{0}\right)\right\} \cdot \nabla \phi(x) d x=0
$$

for any $\phi \in C_{0}^{\infty}(\Omega)$. This implies that $\bar{G}_{p-2}\left(\cdot, y_{0}\right)$ is even with respect to $x_{1}$. By induction, we obtain the result.

Lemma 4.3 Assume $\Omega$ is symmetric with respect to the hyperplane $\left\{x_{1}=0\right\}$ and let $g_{k}(k=0,1,2, \cdots, p-1)$ be odd functions with respect to $x_{1}$. Then, the unique solution $u$ of the problem

$$
\left\{\begin{array}{l}
(-\Delta)^{p} u=0 \quad \text { in } \Omega \\
(-\Delta)^{k} u=g_{k} \quad \text { on } \partial \Omega(k=0,1, \cdots p-1)
\end{array}\right.
$$

is also an odd function with respect to $x_{1}$.

Proof. For $x=\left(x_{1}, x^{\prime}\right) \in \bar{\Omega}$, let us denote $x^{*}=\left(-x_{1}, x^{\prime}\right)$. By the symmetry, we see $x^{*}$ is also a point in $\bar{\Omega}$. Define $v(x)=-u\left(x^{*}\right)$ for $x \in \bar{\Omega}$. Then, by the oddness of $g_{k}$, we obtain

$$
\begin{aligned}
& (-\Delta)^{p} v(x)=-\left((-\Delta)^{p} u\right)\left(x^{*}\right)=0, \quad x \in \Omega, \\
& (-\Delta)^{k} v(x)=-\left((-\Delta)^{k} u\right)\left(x^{*}\right)=-g_{k}\left(x^{*}\right)=g_{k}(x)=(-\Delta)^{k} u(x), \quad x \in \partial \Omega,
\end{aligned}
$$

for $k=0,1, \cdots p-1$. That is, $v$ is also a solution of (4.1). Therefore by the uniqueness of the solution, we obtain that $v=u$, which proves the lemma.

Now, we prove Theorem 4.1.

\section{Proof of Theorem 4.1.}

By Lemma 4.2 , we see that $\left(\frac{\partial \bar{G}_{k}}{\partial x_{1}}\right)\left(\cdot, y_{0}\right)$ is an odd function with respect to $x_{1}$ for $k=0,1, \cdots, p-1$. Now, let $u$ be the unique solution of the problem

$$
\left\{\begin{aligned}
(-\Delta)^{p} u & =0 \quad \text { in } \Omega, \\
(-\Delta)^{k} u & =-\left(\frac{\partial \bar{G}_{k}}{\partial x_{1}}\right)\left(\cdot, y_{0}\right) \quad \text { on } \partial \Omega(k=0,1, \cdots p-1) .
\end{aligned}\right.
$$

By Lemma 4.3, we confirm that $u$ is also odd in $x_{1}$. Therefore, we have $u \equiv 0$ on the hyperplane $\left\{x_{1}=0\right\}$, which implies

$$
\left(\frac{\partial u}{\partial x_{j}}\right)\left(y_{0}\right)=0 \quad \text { for } j=2,3, \cdots, N \text {. }
$$


Note that the same oddness holds for $\bar{u}_{k}(x)=(-\Delta)^{k} u(x)$ for $k=0,1, \cdots, p-$ 1. We see $\bar{u}_{k}$ satisfies

$$
\left\{\begin{array}{l}
-\Delta \bar{u}_{k}=\bar{u}_{k+1} \quad \text { in } \Omega,(k=0,1, \cdots, p-2) \\
-\Delta \bar{u}_{p-1}=0 \text { in } \Omega, \\
\bar{u}_{k}=-\left(\frac{\partial \bar{G}_{k}}{\partial x_{1}}\right)\left(\cdot, y_{0}\right) \quad \text { on } \partial \Omega, \quad(k=0,1, \cdots, p-1) .
\end{array}\right.
$$

Recall $\bar{G}_{k}\left(x, y_{0}\right)>0$ for $x \in \Omega$ and $\bar{G}_{k}\left(x, y_{0}\right)=0$ for $x \in \partial \Omega$ for any $k \in\{0,1, \cdots, p-1\}$. By the assumption $(\mathrm{H} 2)$, we have $\left(\frac{\partial \bar{G}_{k}}{\partial x_{1}}\right)\left(\cdot, y_{0}\right) \geq 0$ on $\left\{x_{1}<0\right\} \cap \partial \Omega$. Also $\bar{u}_{k} \equiv 0$ on $\Omega \cap\left\{x_{1}=0\right\}$ by the oddness of $\bar{u}_{k}$ in $x_{1}$. Then the maximum principle applied to the cooperative system (4.4) on the domain $\Omega \cap\left\{x_{1}<0\right\}$ implies that $\bar{u}_{k}(x)<0$ for $x \in \Omega \cap\left\{x_{1}<0\right\}$ for any $k=0,1, \cdots, p-1$. By applying Hopf lemma in the domain $\Omega \cap\left\{x_{1}<0\right\}$, we also have $\left(\frac{\partial \bar{u}_{k}}{\partial x_{1}}\right)\left(y_{0}\right)>0$ for all $k=0,1, \cdots, p-1$. In particular, we have $u(x)<0$ in $\Omega \cap\left\{x_{1}<0\right\}$ and

$$
\left(\frac{\partial u}{\partial x_{1}}\right)\left(y_{0}\right)>0
$$

On the other hand, by Green's representation formula (2.3), we see the unique solution of (4.2) can be written as

$$
u(y)=\sum_{k=1}^{p} \int_{\partial \Omega}\left(\frac{\partial \bar{G}_{k-1}(x, y)}{\partial \nu_{x}}\right)\left(\frac{\partial \bar{G}_{p-k}\left(x, y_{0}\right)}{\partial x_{1}}\right) d s_{x} .
$$

Differentiating both sides with respect to $y_{j}$ leads to

$$
\frac{\partial u}{\partial y_{j}}(y)=\sum_{k=1}^{p} \int_{\partial \Omega} \frac{\partial}{\partial y_{j}}\left(\frac{\partial \bar{G}_{k-1}(x, y)}{\partial \nu_{x}}\right)\left(\frac{\partial \bar{G}_{p-k}\left(x, y_{0}\right)}{\partial x_{1}}\right) d s_{x} .
$$

Now, compared this to the formula (3.4) in Theorem 3.1:

$$
\frac{1}{2} \frac{\partial^{2} R}{\partial y_{1} \partial y_{j}}(y)=\sum_{k=1}^{p} \int_{\partial \Omega} \frac{\partial}{\partial y_{j}}\left(\frac{\partial \bar{G}_{k-1}(x, y)}{\partial \nu_{x}}\right)\left(\frac{\partial \bar{G}_{p-k}(x, y)}{\partial x_{1}}\right) d s_{x},
$$

and using (4.3), (4.5), we confirm that

$$
\begin{aligned}
& \frac{1}{2}\left(\frac{\partial^{2} R}{\partial y_{1} \partial y_{j}}\right)\left(y_{0}\right)=\left(\frac{\partial u}{\partial y_{j}}\right)\left(y_{0}\right)=0,(j=2, \cdots, N), \\
& \frac{1}{2}\left(\frac{\partial^{2} R}{\partial y_{1}^{2}}\right)\left(y_{0}\right)=\left(\frac{\partial u}{\partial y_{1}}\right)\left(y_{0}\right)>0 .
\end{aligned}
$$


By changing $x_{1}$-axis to another one, we obtain the desired result.

\section{Acknowledgement.}

Part of this work was done while the author visited S.S. Chern Institute of Mathematics (CIM) at Nankai Universiy, Tianjin. The author would like to thank the hospitality of CIM. Part of this work was supported by JSPS Grant-in-Aid for Scientic Research (Kakenhi) (B), No. 23340038.

\section{References}

[1] H. Brezis, and L.A. Peletier: Asymptotics for elliptic equations involving critical growth, Partial differential equations and calculus of variations, Vol.1 ,vol. 1 of Progress. Nonlinear Differential Equations Appl. Birkhüser Boston, Boston, MA, (1989) 149-192.

[2] L. A. Caffarelli, and A. Friedman: Convexity of solutions of semilinear elliptic equations, Duke Math. J. 52 (1985) no.2, 431-456.

[3] P. Cardaliaguet, and R. Tahraoui: On the strict concavity of the harmonic radius in dimension $N \geq 3$, J. Math. Pures Appl. (9) 81 (2002) no.3, 223-240.

[4] K-S. Chou, and D. Geng: Asymptotics of positive solutions for a biharmonic equation involving critical exponent, Diff. Int. Eq. 13 (2000) 921-940.

[5] F. Gazzola, H. C. Grunau, and G. Sweers: Polyharmonic Boundary Value Problems, Lecture Notes in Mathematics, 1991. Springer-Verlag, Berlin, (2010)

[6] B. Gidas, W. M. Ni, and L. Nirenberg: Symmetry and related properties via the maximum principle, Comm. Math. Phys. 68 (1979) 209-243.

[7] M. Grossi: On the nondegeneracy of the critical points of the Robin function in symmetric domains, C.R. Acad. Sci. Paris, Ser. I 335 (2002) 157-160.

[8] E. Mitidieri: A Rellich type identity and applications, Comm. Partial Differential Equations, 18 (1993) no.1-2, 125-151. 
[9] P. Pucci, and J. Serrin: A general variational identity, Indiana Univ. Math. J. 35 (1986) no.3, 681-703.

[10] X. Ren, and J.-C. Wei: On a two-dimensional elliptic problem with large exponent in nonlinearity, Trans. Amer. Math. Soc. 343 (1994) no.2, 749763.

[11] F. Takahashi: Asymptotic behavior of least energy solutions to a fourdimensional biharmonic semilinear problem, Osaka J. Math., 42 (2005) no.3, 633-651. 